[DOI: 10.24214/jecet.A.10.2.11223.]

Juurnal of Enviranmental Science, Computer Science and Engineering \& Technology

An International Peer Review E-3 Journal of Sciences and Technology

Available online at www.jecet.org

Section A: Environmental Science

Research Article

\title{
Gestion des déchets ménagers dans les trois communes de la ville de Kindu, en RDC.
}

\author{
Victor Amuri Abasi ${ }^{1}$, Défi Amuri Assani², Gloire Mukaku Kazadi², \\ Lingot BULEDI AMURI ${ }^{2}$
}

${ }^{1}$ Institut Supérieur de techniques Appliquées de Kindu (RDC)

${ }^{2}$ Université de Kindu. Faculté de Sciences Agronomiques. Département de Zootechnie, Gestion des Ressources Naturelles (GRN) et de Phytotechnie. BP 122 Kindu (RDC).

Reçu: 31 Janvier 2021; Révisé: 19 Février 2021; Accepté: 03 Mars 2021

\begin{abstract}
Résumé : Une enquête a été réalisée dans la ville de Kindu, spécifiquement dans la commune de Kasuku à partir du Mois de Juin au mois de Décembre 2020.La présente étude consistait à faire une analyse sur la problématique de la gestion des ordures ménagères dans la commune précitée. Ce travail poursuit comme objectif global d'étudier les problèmes majeurs liés à la gestion des ordures ménagères dans commune de Kasuku, afin de contribuer à une gestion durable des celles-ci. Pour y parvenir, nous nous sommes servis des méthodes documentaires appuyées par celles d'enquêtes par questionnement direct. Ainsi les résultats ressortis de nos investigations ont montrés que la grande partie de nos enquêtés de la commune de Kasuku possède des poubelles provisoires pour les stockages des leurs déchets. Les résultats obtenus ont montré que 73,3\% de ménages possèdent des poubelles des stockages des ordures mais ces ordures ou déchets ne sont pas triés, 49,3\% des ménages mélangent leurs ordures bio au non dégradables. Quant aux méfaits de la mauvaise gestion des ordures ménagères, les résultats obtenus montrent que 66,6\% des ménages sont conscients de savoir que les conséquences qui sont liées à la mauvaise gestion des ordures ou déchets ménagers dans le cadre de vie de la population. Celles-ci sont jetées dans plusieurs endroits mais surtout dans les cours d'eau à une proposition de $26,7 \%$ et dans une fosse dans le quartier $25,3 \% ; 21 \%$ dans les rues ; $16,7 \%$ à la structure de Mairie et $10 \%$ aux poubelles publiques.
\end{abstract}


Mots clés : Gestion, ordures, ménagère, Kindu, Kasuku

\section{INTRODUCTION}

La gestion des déchets ménagers solides reste un grand défi pour les municipalités Africaines de manière générale, notamment pour celles de la R.D.Congo et celle de Kindu en particulier. Celles-ci rencontrent d'importantes difficultés pour assumer correctement ce service, alors que les lois sur la décentralisation leur ont conféré cette responsabilité. Ces municipalités sont confrontées à une forte croissance de la population, ainsi qu'à une évolution du monde de consommation qui se traduit par une augmentation de déchets ${ }^{[1]}$.

L'urbanisation et l'essor économique contribuent à l'amélioration des conditions de vie de l'individu, mais s'accompagnent chaque fois d'une pollution de diverses natures nécessitant des mesures appropriées pour la neutraliser. D'ailleurs, les plus importantes des pollutions sont d'origine industrielle, commerciale, hospitalière, agricole ainsi que celles qui sont issues de la vie domestique appelées déchets ménagers ou encore ordures ménagères. La pollution engendrée par ce genre de résidu est un problème très préoccupant et il est devenu un sujet d'actualité parce que les détritus délaissés n'importe où et n'importe comment constituent une véritable menace de notre environnement ${ }^{[2]}$.

Les villes des pays en développement font face à un important problème de gestion de leurs déchets. Au-delà des difficultés techniques et financières qui sont souvent évoquées, la mauvaise connaissance du gisement des déchets et de ses caractéristiques est un handicap important pour planifier leur gestion ${ }^{[3]}$.

Cette situation, dont les effets sont visibles par tous, engendre des nuisances importantes pour les habitants et a des conséquences néfastes sur la santé des populations, sur l'environnement et sur les ressources naturelles ${ }^{[4]}$.

La gestion des déchets doit s'inscrire désormais dans la perspective d'un développement durable dont les principes de base mettent en avant un environnement viable (coûts de dégradation de l'environnement), un maintien du capital naturel (rejets éco compatibles) et la biodiversité ${ }^{[5]}$

En effet, chaque jour, les ménages rejettent des tonnes des déchets, reflets de la consommation courante. Il s'agit de nourriture ou de produit de la vie quotidienne, à cela s'ajoute les déchets assimilables aux ordures ménagers ${ }^{[6]}$.

La population joue un rôle principal et est considérée comme un facteur important de la dégradation de l'environnement. L'accroissement de la population mondiale pour les années à venir suscite dès lors des inquiétudes sur l'état de l'environnement. La population mondiale connait une forte croissance et atteindra 9,2 milliards d'habitants en 2075 contre 6,4 milliards actuellement ${ }^{[7]}$.

Le problème de déchets est un phénomène de société et plus que jamais celui-ci est confronté à la production et à la gestion de ses déchets. Conséquence de nos modes de vie, les déchets ménagers ne cessent de croitre et leur rejet dans l'environnement devient un problème, ainsi nous ne vivons plus seulement dans une société de production et de consommation mais aussi dans une société de déjection. Le besoin de consommation s'accompagne du besoin de rejeter les résidus, de préférence sans se soucier de ce qu'ils adviendront puisqu'ils sont connus pour être sales et embarrassants ${ }^{[8]}$.

La croissance économique et démographique, si elles s'accompagnent d'une exploitation accélérée des ressources naturelles, son corollaire, la production des déchets est par définition inévitable. Plus qu'une question théorique, cela pose un énorme problème logique et économique aux administrateurs des villes surtout dans les pays en développement. 
Face à cette situation, les hypothèses suivantes sont avancées.

- La production de déchet solide ménager (DSM) augmenterait partout dans le monde à cause de modifications des styles de vie, surtout dérivés de l'industrialisation à travers l'incitation à la consommation, l'augmentation du pouvoir d'achat et l'industrialisation des produits grâce aux emballages qui ne servent plus à d'autres utilisations.

- L'explosion démographique, l'accroissement des activités urbaines, l'insuffisance des ressources financières des pouvoirs publics au niveau local et la pauvreté de la population seraient autant des facteurs qui expliqueraient la présence des déchets le long des grandes artères des villes de l'Afrique sub-saharienne.

Cette recherche a été menée dans l'objectif global d'étudier les problèmes majeurs liés à la gestion des ordures ménagères à Kindu dans la commune de Kasuku afin de contribuer à une gestion durable des celles-ci et mettre à l'abri la population toute qui y habite.

D’une manière spécifique, cette étude poursuit les objectifs suivants :

- Déterminer les causes de l'accroissement des ordures ménagères dans la commune Kasuku ;

- Evaluer les conséquences de l'accroissement des ordures ménagères dans la commune Kasuku ;

- Proposer les pistes des solutions pour réduire leurs conséquences, afin d'avoir une gestion durable des ordures ménagères dans cette commune.

Ces genres d'études bien que d'actualité mais restent encore assez rares dans la ville Kindu. C'est dans cette optique qu'est né le souci de mener une telle recherche allant dans le sens de déterminer les causes et conséquences de la mauvaise gestion des ordures ménagères.

\section{MATERIEL ET METHODES}

2.1. Présentation du milieu d'étude : La ville de Kindu est une agglomération urbanorurale qui est située à l'Est de la RDC avec les coordonnées géographiques suivantes : latitude Sud est de $2^{\circ} 56^{\prime}$ et $37^{\prime \prime}$, longitude est de $25^{\circ} 55$ et $20^{\prime \prime}$, l'altitude est de $464 \mathrm{~m}$. La saison pluvieuse intervient de mi-aout à mi-mai, la température moyenne annuelle de $25^{\circ}$ à $27^{\circ} \mathrm{c}$ et la moyenne de précipitation s'est élevée à $1650 \mathrm{~mm}$ d'eau.

La commune de Kasuku est celle qui a concerné notre champs d'étude, elle est limitée au :

Au Nord: par une droite partant du P.K 5 rive gauche du fleuve Congo via le point nord gauche de la piste de l'Aéroport National de Kindu jusqu'au cimetière de la route Lokandu.

$>\boldsymbol{A u}$ sud : croisement route Lwama et Lokandu jusqu'à l'intersection de la rivière Kapondjo. De là, jusqu'au confluent avec la rivière Mikelenge jusqu'à son embouchure avec le fleuve Congo.

$>\boldsymbol{A}$ l'Est : la rive gauche du fleuve du PK5 jusqu'à l'embouchure de la rivière Mikelenge.

A l'Ouest: Axe route Lokandu-Lwama via cimetière jusqu'au coin Nord de la piste de l'Aéroport (Rapport annuel 2019 commune Kasuku).

* Coordonnées géographiques (latitude, longitude): Les coordonnées géographiques de la commune de Kasuku sont les mêmes que celles de la ville de Kindu.

- $\quad 2^{\circ} 57^{\prime}$ de latitude sud 
- $\quad 25^{\circ} 27^{\prime}$ de latitude Est

Superficie : $30 \mathrm{~km}^{2}$

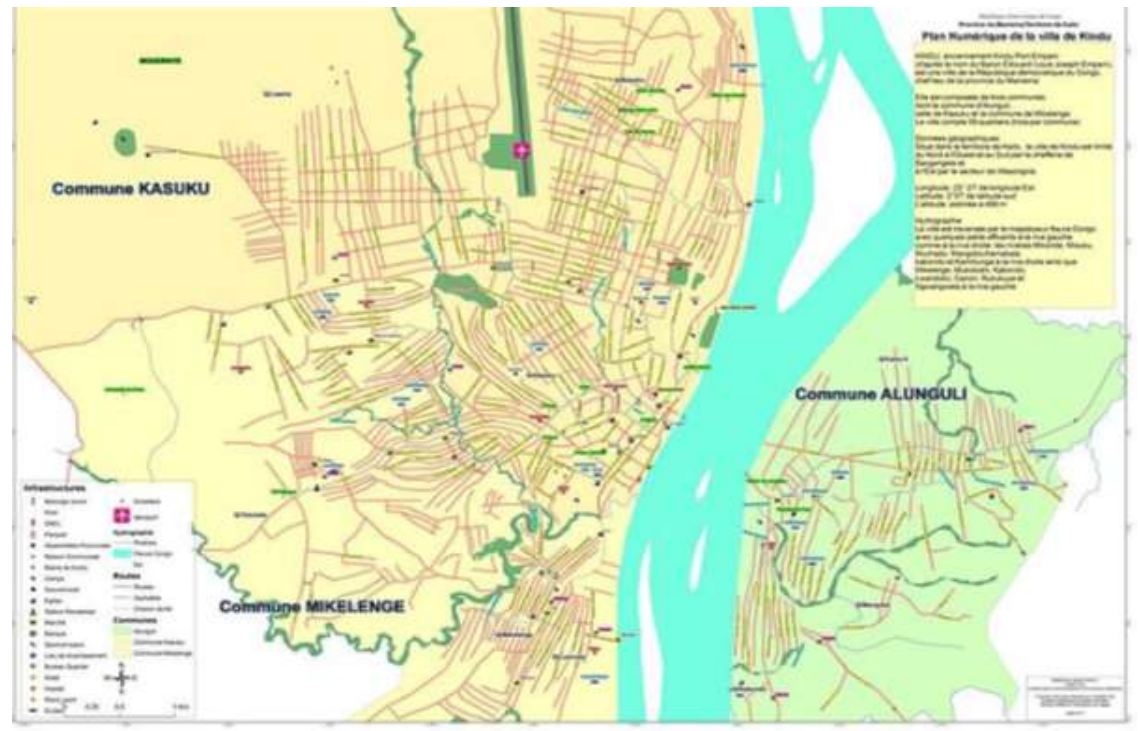

Fig. 1 : La ville de Kindu

- Données géographiques

\section{$>$ Type de climat}

En principe la Commune de Kasuku a deux saisons. La saison sèche et la saison de pluie. Il se fait malheureusement qu'au courant de l'année 2019-2020, il y a eu une véritable perturbation sur toute l'étendue de la commune de Kasuku en particulier et de la ville de Kindu en Général. Surtout la pluie a débordé la vase à tel enseigne que les agriculteurs ne savent pas à quel saint vouer ${ }^{[9]}$.

\section{La nature du sol}

Le sol reste toujours argilo-sablonneux soit sablo-argileux.

- $\quad$ Le relief du sol

Son relief est très riche.

- $\quad$ Renseignement sur le sous-sol

Le besoin de construction se fait toujours sentir et que la production par concassage des pierres dans des concessions environnant les habitants reste d'actualité ${ }^{[10]}$.

\section{- La végétation dominante}

Les quartiers Basoko et Lwama sont Urbano-ruraux, les populations qui habitent les communes sœurs rurales sont soumises au paiement des différentes exigences par les autochtones Bangengele et Wasongola.

\section{II.1.8. Hydrographie}

Elle dominée par le fleuve Congo qui constitue la limite naturelle avec la commune sœur d'Alunguli, tandis que les rivières Mikelenge et Ngwangwata, Kano et Kabondo sont d'autres ruisseaux qui coulent à travers la commune de Kasuku ${ }^{[9]}$ 


\section{II.1.9. Population}

Constituée d'une Ethnie Bantu, les nilotiques viennent temporairement pour vendre leurs vaches, mais d'autres ont trouvé domicile dans la commune de Kasuku. Le pygmoïde est presqu'inexistant. Les tribus dominantes sont concentrées dans trois quartiers de la commune :

$\checkmark$ Pour le quartier Basoko l'on trouve les Metuku, Zimba, Bangubangu, et les Basongola,

$\checkmark$ Pour le quartier Kasuku l'on trouve les Kusu, Tetela, Zimba, Bangubangu, Rega, Nonda+Kasenga, Zura, Buyu.

$\checkmark$ Pour le quartier Lwama l'on trouve les Langa, Ngengele, et aussi les Zimba.

Ces tribus sont regroupées en mutuelle des ressortissants.

$\checkmark$ Les territoires de Lubutu et Punia par la mutuelle Ekita

$\checkmark \quad$ Les territoires de Kailo par la mutuelle : Lubunga ;

$\checkmark$ Le territoire de Pangi par la mutuelle : Lusu;

$\checkmark$ Le territoire de Kabambare pour la mutuelle : MURESKA ;

$\checkmark \quad$ Le territoire de KASONGO pour la mutuelle : AMUKA ;

$\checkmark \quad$ Le territoire de Kibombo pour la mutuelle : ADERKISAL ${ }^{[9]}$.

2.2. Materiel: Pour conduire notre étude, nous avons fait recours aux matériels suivants :

$\checkmark$ Un questionnaire d'enquête pré élaboré qui nous a permis de collecter les informations auprès des ménages ;

$\checkmark$ Stylo, crayon, gamme, marqueur et latte;

$\checkmark$ Rame de papier duplicateur, format A4;

$\checkmark$ Calculatrice scientifique ;

$\checkmark$ Un appareil photo qui nous a permis d'acquérir les images dans les endroits pollués.

2.3. Methodes: Une enquête a été menée dans la ville de Kindu précisément dans la Commune de Kasuku, du 22 janvier au 22 avril 2020, auprès de 300 ménages.

Méthodes et techniques d'enquête: Pour obtenir les résultats, nous nous sommes servi de méthode d'enquête, la méthode statistique et la méthode analytique.

En effet ces méthodes étaient soutenues par les techniques ci-après: recherche documentaire, l'observation, l'interview directe, le choix des enquêtés et les logiciels Word et excelle.

2.3.1. Recherche documentaire: La recherche documentaire est une étape de travail à réaliser avant de se lancer dans une étude empirique. Elle permet de collecter des données informatives grâce à l'étude de documents officiels.

A partir d'un sujet d'enquête connu, la recherche documentaire revient à chercher et identifier des documents issus de sources fiables. Les informations récoltées seront utiles pour développer ses connaissances sur le sujet étudié ${ }^{[11]}$.Des données ont été collectées dans les différents ouvrages disponibles dans les bibliothèques et sur l'internet. Cette recherche documentaire a porté sur les ouvrages, rapport de stage, mémoire, thèse etc. abordant la problématique de la gestion de l'environnement et de la gestion des ordures ménagères. 
II.3 .2. Observation du terrain: Des visites effectuées sur terrain ont permis d'avoir une réalité sur l'état d'insalubrité de notre milieu d'étude.

II.3.3. Enquêtes: Une enquête est une opération qui a pour but la découverte de faits, l'amélioration des connaissances ou la résolution des problèmes. L'enquête réalisée a permis d'approfondir notre recherche et d'apporter des éléments des réponses à certaines questions liées à la gestion des ordures ménagères.

II.3.4. Questionnaire d'enquête: Cette technique a permis des recueillir les informations auprès de la population. Il a été adressé dans un premier temps aux populations de Kasuku dans le but d'obtenir des informations sur la façon de gérer les déchets et comprendre leurs impacts sur la santé et sur l'environnement. Dans un second temps, il a été adressé aux associations pour la protection de l'environnement (A.P.E) pour comprendre aussi leur système de collecte et leurs problèmes. Pour ce faire, nous avons utilisé un questionnaire adressé aux chefs de ménages. Ce questionnaire porte sur la gestion des ordures ménagères.

1. Cibles: L'enquête par questionnaire a visé les chefs de ménages, mais en grande partie, ce sont les femmes que nous rencontrions parce que ce sont elles qui s'occupent des travaux ménagers. Le choix des ménages était aux hasards.

2. Taille de l'échantillon: Pour bien faire ce travail nous avons pris un échantillon de 300 ménages (personnes) qui ont été choisis au hasard. Les échantillons ont été répartis dans trois (3) quartiers en raison de 100 ménages par quartier. Les informations enregistrées des enquêtes sur terrain ont fait l'objet d'un traitement manuel et informatique dans le but de garantir la fiabilité des résultats.

\section{II.3.5. Traitement des données}

a) Logiciel utilisé : Excelle et Word

b) Analyse statistique

Les réponses fournies par les enquêtés furent analysées de manière statistique. Ça nous a permis d'obtenir des différentes fréquences qui ont été converties en pourcentage. La formule qui était utilisée est la suivante :

Formule $: P=\frac{F O}{N} x 100$

$\mathrm{P}(\%)=$ Pourcentage

$\mathrm{F}^{\mathrm{o}}=$ Fréquence observée

$\mathrm{n}=$ Fréquence totale

$\sum=$ Sommation $^{[12]}$

\section{Présentation, Interprétation et Discussion des résultats:}

Cette partie sera présentée en cinq points exposant le profil des enquêtés, le type d'ordures produites, moyen d'évacuation, site de stockage et de destruction des déchets, en fin la conséquence de la mauvaise gestion des déchets.

\section{Profil des enquêtés}


Tableau 1 : présentation de sexe, tranche d'âge, profession et taille de ménages des enquêté

\begin{tabular}{lllllll}
\hline Caractéristiques & Modalités & Basoko & Kasuku & Lwama & Moyenne & P \% \\
\hline Sexe de l'enquêté & Masculin & 48 & 44 & 42 & 44,6 & 44,6 \\
& Féminin & 52 & 56 & 58 & 53,3 & 55,3 \\
Tranche d'âge & $<$ à 20 & 10 & 24 & 18 & 17,3 & 17,3 \\
& De 20 à 30 & 42 & 30 & 46 & 39,3 & 39,3 \\
& De 30 à 50 & 36 & 32 & 26 & 31,3 & 31,3 \\
& $>$ à 50 & 12 & 14 & 10 & 12 & 12 \\
Profession & & & & & & \\
& Fonctionnaires & 12 & 28 & 14 & 18 & 18 \\
& Commerçants & 6 & 18 & 12 & 12 & 12 \\
& Cultivateurs & 16 & 0 & 4 & 6,6 & 6,6 \\
& Elèves/Etudiants & 32 & 28 & 40 & 33,3 & 33,3 \\
& Femmes ménagères & 34 & 26 & 30 & 30 & 30 \\
& $<5$ & 18 & 56 & 20 & 31 & 31 \\
& 5 àille des ménages & 64 & 34 & 66 & 54,6 & 54,6 \\
& Plus de 10 & 18 & 10 & 14 & 14 & 14 \\
\hline
\end{tabular}

Il ressort de ce tableau que $55,3 \%$ de nos enquêtés sont de sexe féminin par contre $44,7 \%$ d'entre eux sont de sexe masculin. Les personnes âgées de 20 à 30 ans priment avec 39,3\%, suivies de celles variant entre 30 à 50 ans avec 31,3\%, suivies des personnes de moins de 20 ans présentant 17,3\% et de celles de plus de 50 ans qui présentent $12 \%$. Pour ce qui est de la profession, 33,3\% des répondants sont des élevés et/ou étudiants, $30 \%$ d'eux sont des femmes ménagères, $18 \%$ de nos enquêtés sont des fonctionnaires, $12 \%$ des enquêtés représentent les commerçants et, enfin $6,7 \%$ des enquêtés représentent les cultivateurs. les ménages dont leur nombre varient de 5 à 10 personnes sont nombreux avec une proportion de 54,7\%, suivis des ménages qui varient de 1 à 5 personnes avec 31 , $3 \%$ et ceux de plus de 10 personnes avec $14 \%$.

\section{Production des ordures}

Le Tableau $\mathbf{N}^{\circ} 2$ : Répartition de nos enquêtés selon les types de déchets produits

\begin{tabular}{lll}
\hline Type & Fréquence observée $\left(\mathbf{F}^{\circ}\right)$ & Pourcentage \\
\hline Biodégradable & 122 & 40,6 \\
Non biodégradable & 30 & 10 \\
Tous le deux & 148 & 49,3 \\
& & \\
\hline Total & $\mathbf{3 0 0}$ & $\mathbf{9 9 , 9}$ \\
\hline
\end{tabular}


Dans ce tableau, il se dégage 49,3\% des ménages des enquêtés produisent le deux types déchets cités dans le tableau 2 ci-haut, $40,6 \%$ de nos répondants produisent des déchets biodégradables et $10 \%$ des ménages produisent des déchets non biodégradables.

\section{Disponibilité d'un lieu d'évacuation des déchets}

Tableau $\mathrm{N}^{\circ} 3$ : Connaissance sur la possession de poubelle de stockage des ordures dans les ménages de Kasuku

\begin{tabular}{lll}
\hline Poubelle & Fréquence observée $\left(\mathbf{F}^{\circ}\right)$ & Pourcentage \\
\hline Oui & 220 & 73,3 \\
Non & 80 & 26,7 \\
\hline Total & $\mathbf{3 0 0}$ & $\mathbf{1 0 0}$ \\
\hline
\end{tabular}

Le tableau ci-haut démontre que 73,3\% des ménages enquêtés des ménages enquêtés n'utilisent pas des poubelles de stockage telles que sceau, sac, etc., mais ils exposent seulement leurs ordures ménagères directement sur le sol, dans le trou creusé comme leur destination possèdent des cantine, sacs, carton dans lesquels sont stockées leurs ordures ménagères, et seulement $26,7 \%$ finale.

Tableau $\mathbf{N}^{\circ}$ 4: Connaissance sur les types de poubelles provisoires utilisées par la population de

$$
\text { Kasuku : }
$$

\begin{tabular}{lll}
\hline Type de poubelle & Fréquence observée $\left(\mathbf{F}^{\circ}\right)$ & Pourcentage \\
\hline Trou & 46 & 15,3 \\
Sac & 66 & 22 \\
Carton & 48 & 12,7 \\
Cantine & 104 & 34,7 \\
Autre (caniveau, directement aux rues) & 46 & 15,3 \\
\hline Total & $\mathbf{3 0 0}$ & $\mathbf{1 0 0}$ \\
\hline
\end{tabular}

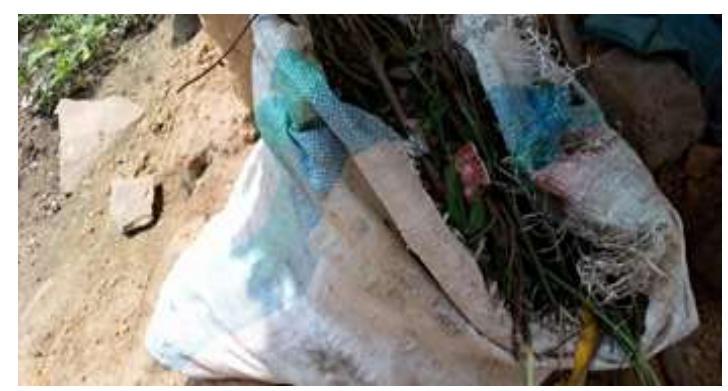

Fig. 1: Poubelles à côté des ménages

Il découle de ce tableau $\mathrm{N}^{\circ} 5$ ci-haut que les ménages qui stockent leurs ordures ménagères dans des cantine (sceau) sont plus nombreux (52 ménages) avec une proportion de 34,6\%, suivisdeceux-là qui stockent dans les sacs avec $22 \%$, dans des trous y compris ceux jettent dans les différentes endroits cités dans le tableau(par exemple dans les caniveaux, rues etc.) avec respectivement chacun $15,3 \%$ et suivis enfin des ménages qui gardent les leurs dans des cartons avec une proportion de $12,7 \%$. 
Il nous revient de signaler que tous ces types de poubelles évoquées ci-haut sont provisoires.

\section{La destination finale des poubelles provisoires}

Tableau $\mathbf{N}^{\circ} 5$ : L'endroit où sont jetées les ordures ménagères de Kasuku après avoir été gardées aux ménages provisoirement

\begin{tabular}{llll}
\hline Destination ou endroit & $\begin{array}{l}\text { Fréquence } \\
\text { observée }\left(\mathbf{F}^{\circ}\right)\end{array}$ & Pourcentage \\
\hline - & Poubelle publique & 30 & 10 \\
- & Une fosse dans le quartier & 76 & 25,3 \\
- & Structure de Maitre & 50 & 16,7 \\
- & APE & 0 & 0 \\
- $\quad$ Cours d'eau & 80 & 26,7 \\
- Rues & 64 & 21,3 \\
\hline Total & $\mathbf{3 0 0}$ & $\mathbf{1 0 0}$ \\
\hline
\end{tabular}

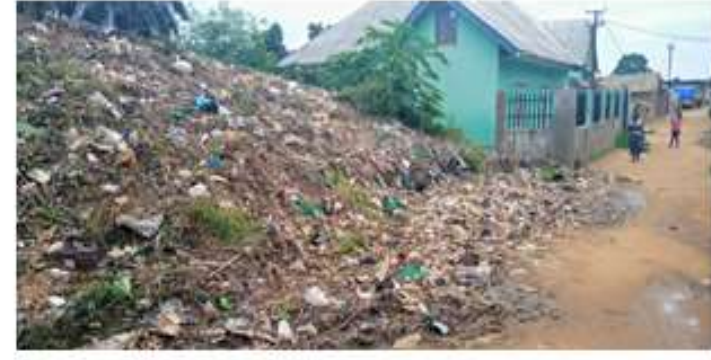

Fig.2: Poubelles à côté des ménages

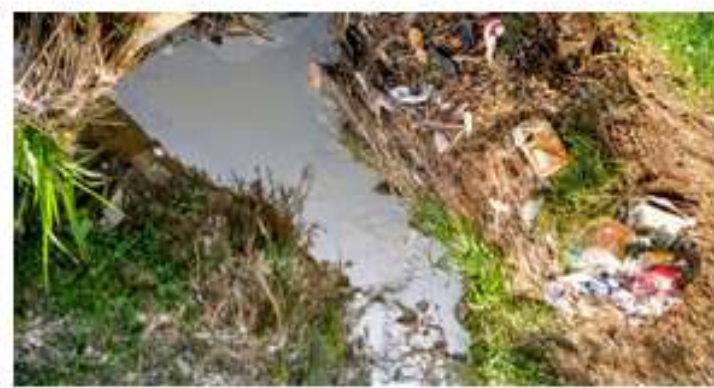

Fig.4: Déchets de poubelles dans les cours d'eau

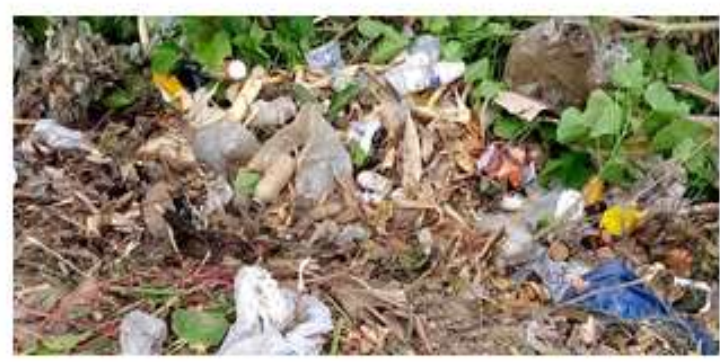

Fig. 3: Les poubelles dans les quartiers

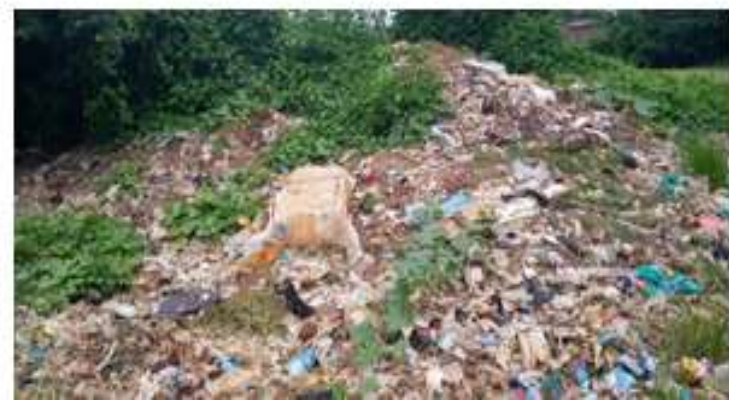

Fig.5: Poubelle Poubelle publique

L'examen de ce tableau N5 5 ci-dessous témoigne sur 150 enquêtés, 40 ménages soit 26,7\% déposent leurs ordures ménagères dans des petits cours d'eau qui traversent leurs blocs, avenues, etc. les autres au fleuve Congo, suivis de ceux qui jettent dans une fosse dans le quartier avec $25,3 \%$; suivis de 21 , $3 \%$ des enquêtés qui jettent leurs ordures ménagères dans les rues, $16,7 \%$ des répondants payent la structure de la Mairie qui passe ramasser leurs ordures dans des différents coins où il déposent, et $10 \%$ des enquêtés jettent leurs ordures dans le poubelles publiques. 
Tableau Nº6 : Moyen de transport utilisé pour l'évacuation des ordures ménagères dans la commune Kasuku

\begin{tabular}{lll}
\hline Moyen & Fréquence observée $\left(\mathbf{F}^{\circ}\right)$ & Pourcentage \\
\hline Vélo & 16 & 5,3 \\
Brouette & 36 & 12 \\
Chariot & 20 & 6,7 \\
Corps humain & 228 & 76 \\
\hline Total & $\mathbf{3 0 0}$ & $\mathbf{1 0 0}$ \\
\hline
\end{tabular}
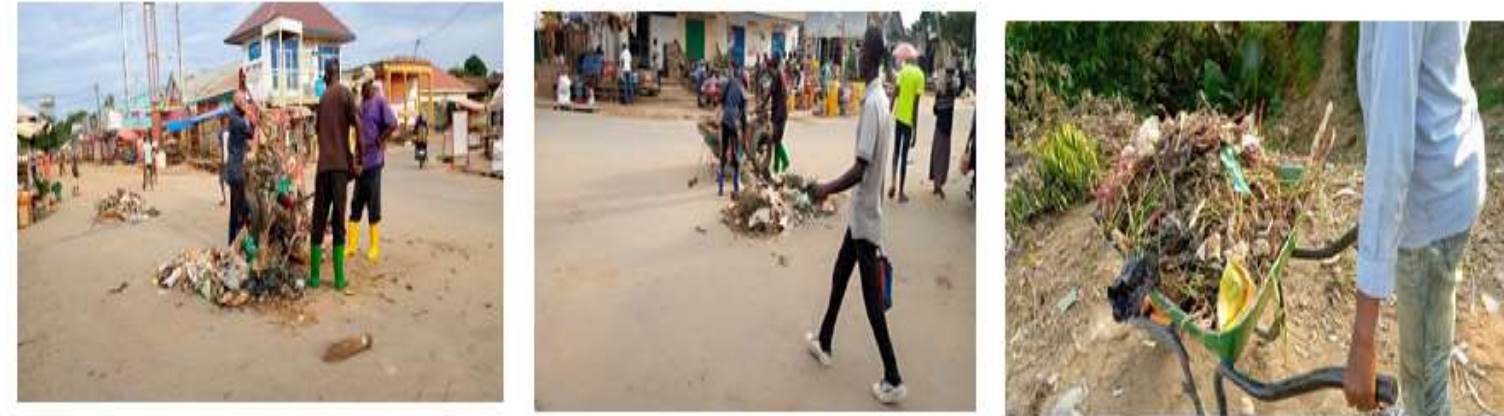

Fig.6: transport des ordures avec (a)la brouette (b) la tête (c) la main

L'étude de ce tableau relève que $76 \%$ des ménages transportent eux-mêmes leurs produits sur la tête et/ou à la main, suivis de ceux qui transportent leurs ordures avec la brouette présentant 12\%, suivis de 6,7\% des ménages qui utilisent le chariot et enfin 5,3\% des ménages transportent leurs ordures par le vélo.

Tableau No7: Rythme d'évacuation des ordures ménagères

\begin{tabular}{lll}
\hline Rythme & Fréquence observée $\left(\mathbf{F}^{\circ}\right)$ & Pourcentage \\
\hline - Chaque jour & 42 & 28 \\
- Une fois/Semaine & 23 & 15,3 \\
- Deux fois/Semaine & 22 & 14,7 \\
- Une ou deux fois/Semaine & 11 & 7,3 \\
- Autre (2, 3,4 Jours,...) & 52 & 34,7 \\
\hline Total & $\mathbf{1 5 0}$ & $\mathbf{1 0 0}$ \\
\hline
\end{tabular}

Il se dégage dans le tableau $\mathrm{N}^{\circ} 7$ ci-haut portant sur les rythmes d'évacuation des ordures que 52 ménages soit $34,7 \%$ évacuent leurs ordures juste après 2,3 ou 4 jours ; 42 ménages des enquêtés soit $28 \%$ évacuent leurs ordures chaque jour ; suivi de 15,3\% soit 23 ménages qui les évacuent une fois par semaine ; $14,7 \%$ évacuent les leurs deux fois par semaine et enfin par semaine et enfin 7,3\% soit 11 ménages des enquêtés évacuent leurs ordures une ou deux fois par semaine selon leurs modes de vie. Il est observé que les ménages qui évacuent leurs déchets chaque jour, deux ou trois jours après sont ceux qui sont plus proches des lieux d'élimination ou de la destination finale, tandis que ceux qui sont éloignés le font une, deux fois la semaine. 


\section{Les conséquences de la mauvaise gestion des ordures ménagères :}

L'examen de secteur 1 ci-dessus témoigne que sur 150 ménages enquêtés, 100 ménages soit 67\% connaissent les méfaits ou conséquences de la mauvaise gestion des ordures ménagères sur la santé humaine et dans leur milieu de vie, tandis que 33\% des enquêtés soit 50 ménages sont ignorants, ne connaissent aucun méfait de la mauvaise gestion des ordures. Parmi les conséquences évoquées, il s'agit notamment l'apport des moustiques anophèles qui causent la malaria, la mauvaise odeur, pollution de l'air qu'ils respirent, du sol ainsi que de l'eau, des blessures, fièvre typhoïde, la diarrhée, etc.

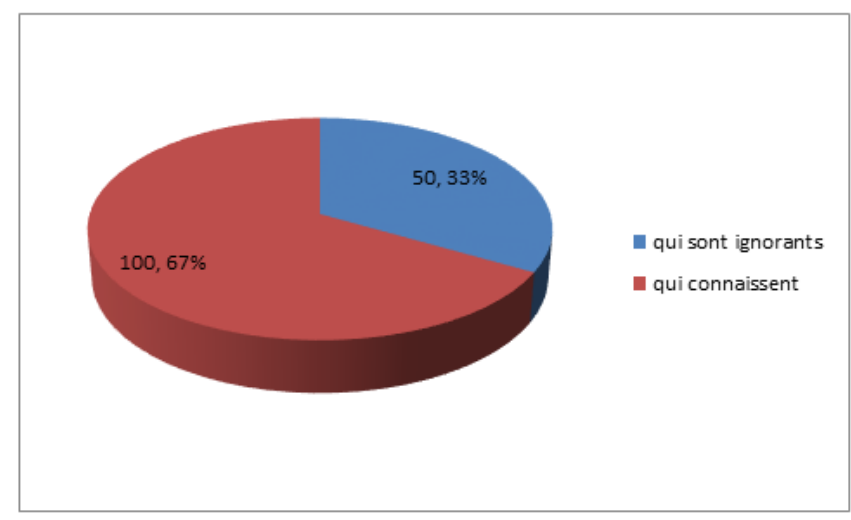

Secteur 1: Les conséquences de la mauvaise gestion des ordures ménagères

\section{REFERENCE}

1. A.Défi, Y. Brahimu, V. Amuri et N. katokolo, Caractérisation morphométrique et commercialisation des œufs de la poule locale (Gallus gallus domesticus L.) à Kindu en République Démocratique du Congo, Revue Africaine d'Environnement et d'Agriculture $2020 ; 3(2), 82-87,87 \mathrm{p}$.

2. L.Y.Mayster et V. Duflon, déchets urbains, nature et caractérisation Suisse, éditions presses polytechniques et universitaires Romandes,1994, 219.P ;

3. Emmanuel et al, évolution des caractéristiques des déchets solides ménagers dans la ville de Yaoundé au Cameroun (1995-2015),2017, ouvrage, $3^{\mathrm{e}}$ page, 16pp ;

4. Ali, problématique de la gestion des déchets ménagères urbains dans la ville de N'djamena, Institut international d'ingénierie, de l'Eau et de l'environnement, mémoire : option: environnement urbain $, 2012,2^{\mathrm{e}}$ page, 55PP. institut international d'ingénierie, l'eau et de l'environnement ;

5. Sidi, méthodologie de caractérisation des déchets ménagers à Nouakchott (Mauritanie), université de Limoges, Thèse,2006, 20e page, 195PP ;

6. GUERMOUD Noreddine et ADDOU Ahmed, étude et caractérisation des déchets ménagers de la ville de Mostaganem (Ouest-Algérie) 2014.

7. J. Véron ,La moitié de la population mondiale vit en ville, population et société $\mathrm{n}^{\circ}$ Appliquée, juin 2007, INED P.1-4 ;) ;

8. C.Florence, le traitement et la gestion des déchets ménagers à la réunion, université de la réunion, thèse, $2007,203^{\mathrm{e}}$ page, $421 \mathrm{PP}$; 
9. Rapport annuel 2019, rapport Annuel de l'administration du territoire. E.T.D/ Mairie de la ville de Kindu, $1^{\text {ère }}$ page, $2^{\mathrm{e}}$ page. $129 \mathrm{PP}$.

10. Rapport annuel 2018, Mairie de Kindu commune Kasuku, ville de Kindu, province du Maniema, RDC, page1, 2, 98PP.

11. Gaspard Claude, 2019, Scribbr, Recherche documentaire méthodologie sur https://WWW.Scribbr.Fr.....Méthodolodie. A jour le 28 Mai 2020 ;

12. Misenga, statistique et biométrie, cours inédit FSA/UNIKI/G3,2015.

* Corresponding Author: Victor Amuri Abasi,

Institut Supérieur de techniques Appliquées de Kindu (RDC)

Online date of publication: 00.03.2021 